\title{
Morbidity and mortality in the surgery arm of EORTC 08941 trial
}

\author{
P. Van Schil, J. Van Meerbeeck, G. Kramer, T. Splinter, C. Legrand, G. Giaccone, \\ C. Manegold and N. van Zandwijk
}

ABSTRACT: Controversial results of surgical treatment after induction chemotherapy, especially in relation to the extent of resection, have previously been reported. Mortality and morbidity were studied in the surgical arm of the European Organisation for Research and Treatment of Cancer (EORTC) 08941 trial.

EORTC 08941 is a multicentre, prospective, randomised, phase-III trial of surgical resection versus radiotherapy in patients with proven stage IIIA-N2 nonsmall cell lung cancer after an objective response to platinum-based induction chemotherapy. Operative results in the 167 patients randomised in the surgical arm are presented within this study. Among these patients, one switched to the radiotherapy arm and 17 patients did not get any protocol treatment or information is not yet available.

Radical resection with negative surgical margins was obtained in 74 patients (49.7\%). In 61

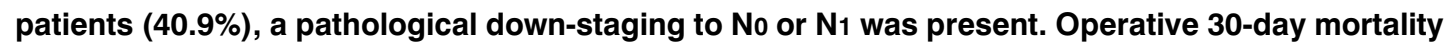
was $4.0 \%$. Post-operative complications were mainly pneumonia, respiratory insufficiency, arrhythmias, air leak, cardiac decompensation, empyema and bronchopleural fistula. In total, $12(8.1 \%)$ patients underwent re-operation due to positive margins, haemothorax, empyema and bronchopleural fistula.

In conclusion, surgical resection after induction chemotherapy in the multicentre European Organisation for Research and Treatment of Cancer trial has yielded acceptable rates of morbidity and mortality.

KEYWORDS: Chemotherapy, lung cancer, morbidity, mortality, neoadjuvant therapy, surgery

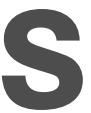
tage IIIA nonsmall cell lung cancer (NSCLC) with involvement of ipsilateral mediastinal or subcarinal nodes (N2) represents a heterogeneous group of patients, ranging from minimal intranodal involvement to extracapsular bulky disease [1]. To date, management of pathologically proven stage IIIA-N2 disease is controversial and the precise role of surgery to obtain local control has not yet been established. Many phase-II, and two smaller phase-III, studies have demonstrated favourable results with induction chemotherapy followed by surgical resection [2]. However, the role of surgical resection versus radiotherapy for optimal local control has not been addressed in these studies. For this reason, large, multicentre phase-III trials were initiated in the USA and Europe to provide a more definitive answer [2, 3]. In the European Organisation for Research and Treatment of Cancer (EORTC) disease were randomised to either surgery or radiotherapy after a response to induction chemotherapy.

Variable results of surgical treatment after induction chemotherapy or chemoradiotherapy have been reported, and high mortality rates after right pneumonectomy are of special concern $[4,5]$. In the present study, the surgical results of the operated patients in EORTC 08941 trial, with special emphasis on mortality, are reported in relation to the extent of resection.

\section{PATIENTS AND METHODS}

The general treatment scheme of the EORTC 08941 trial is depicted in figure 1 and eligibility criteria at registration are given in table 1 . In the study, patients with proven stage IIIA-N2 NSCLC underwent cisplatin-based induction
08941 trial, patients with proven stage IIIA-N2

\section{AFFILIATIONS}

EORTC (European Organisation for Research and Treatment of Cancer), Brussels, Belgium.

CORRESPONDENCE

P. Van Schil

Dept of Thoracic and Vascular Surgery

University Hospital of Antwerp

Wilrijkstraat 10

B-2650 Edegem (Antwerp)

Belgium

Fax: 3238214396

E-mail: paul.van.schil@uza.be

Received:

November 072004

Accepted after revision: April 302005

\section{SUPPORT STATEMENT}

This study was supported by grants 5U10CA11488-24 through 5U10CA11488-34 from the National Cancer Institute (Bethesda, MA, USA). The contents of this study are solely the responsibility of the authors and do not necessarily represent the official views of the National Cancer Institute. This study was reported on behalf of the EORTC Lung Cancer Group. 


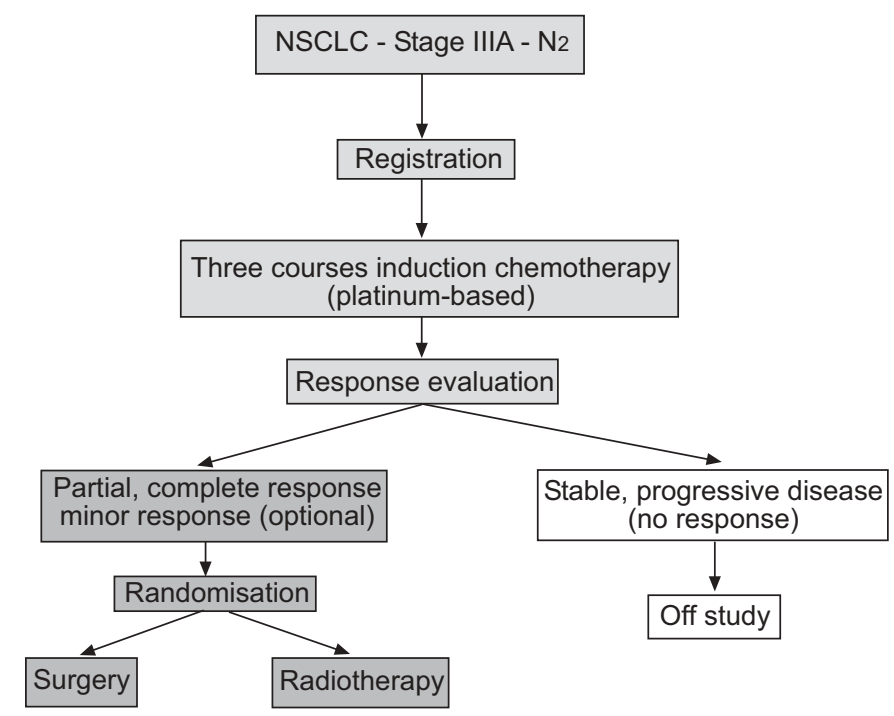

FIGURE 1. General treatment scheme of European Organisation for Research and Treatment of Cancer (EORTC) 08941 trial. NSCLC: nonsmall cell lung cancer.

TABLE $1 \begin{aligned} & \text { Eligibility criteria of European Organisation for } \\ & \text { Research and Treatment of Cancer (EORTC) } \\ & 08941 \text { trial }\end{aligned}$
$\begin{aligned} & \text { Proven clinical stage IIIA-nonsmall cell lung cancer } \\ & \text { Cytological or histological proof of N2 disease }\end{aligned}$
Uni- or bi-dimensional measurable tumour
Irresectable N2 disease
No symptomatic central nervous system involvement
No prior chemotherapy or radiotherapy
No other malignancies
Informed consent

chemotherapy. In case of a response on computed tomography scan of the chest, patients were randomised between surgery and radiotherapy. Patients with progressive disease were included in the study, but not considered for randomisation (off study).

The final aim of surgical treatment in the present trial was complete resection, i.e. radical removal of the primary tumour, hilar and mediastinal lymph nodes. A distinction was made between the following: R0 resection, i.e. complete resection, no macroscopic tumour left behind, negative surgical margins and highest mediastinal node negative on pathological examination; R1, microscopic residual disease; and $\mathrm{R} 2$ resection with macroscopic residual tumour. Post-operative additional radiotherapy was only administered in case of incomplete resection ( $\mathrm{R} 1$ or $\mathrm{R} 2$ ). Staging was carried out according to the most recent tumour, node, metastasis (TNM) classification [6]. Mortality, acute post-surgical events and morbidity data were prospectively collected for each individual patient. The study was performed in centres with a specific interest in thoracic surgery. For this reason, a general outline of complications was provided, but management was left to the local policy of the institution.
Registration of the EORTC 08941 trial closed in December 2002. In total, 333 patients were randomised, 166 to the radiotherapy arm and 167 to the surgical resection group. The latter patients are described in the present study.

At registration, the median age of the patients randomised to surgery was 60 yrs (range 29-78), of which 119 were males and 48 females.

Proof of N2 disease was obtained by cervical mediastinoscopy in 127 patients, anterior mediastinoscopy in 14, video-assisted thoracic surgery in four, thoracotomy in eight and via needle biopsy in 14 patients.

All patients except one had three cycles of platinum-based induction chemotherapy. The regimens used most often were cisplatin-gemcitabine, carboplatin-paclitaxel, cisplatinetoposide and cisplatin-vindesine. Combination chemotherapy with mitomycin, which may be associated with a higher incidence of respiratory complications, was only used in seven patients [7].

The histological types at randomisation were: squamous cell carcinoma in 65 patients (38.9\%); adenocarcinoma in 57 (34.1\%); large cell carcinoma in $41(24.6 \%)$; and mixed cell or other malignant type in four patients $(2.4 \%)$.

Treatment received in the surgical arm was: surgery in 127 patients $(76.0 \%)$; surgery followed by adjuvant radiotherapy in $22(13.2 \%)$; one patient $(0.6 \%)$ switched to the radiotherapy arm; nine patients $(5.4 \%)$ had no treatment according to protocol; and information on treatment actually received was not available for eight patients $(4.8 \%)$. The group of 149 patients who had surgery $(n=127)$ or surgery followed by radiotherapy $(n=22)$ was considered for further analysis.

Regarding the type of surgery, pneumonectomy was performed in 69 patients $(46.3 \%)$, lobectomy or bilobectomy in 58 $(38.9 \%)$, exploratory thoracotomy in $21(14.1 \%)$ and another intervention in one patient $(0.7 \%)$ who underwent remediastinoscopy, which was a protocol violation. As remediastinoscopy remained positive, this patient had no surgical resection.

There were $35(50.7 \%)$ pneumonectomies on the right side, 33 $(47.8 \%)$ on the left side and, for one patient $(1.4 \%)$, the side of pneumonectomy was unknown.

The impact of several baseline factors on the risk of postsurgical morbidity and mortality was investigated using univariate and multivariate (with backward selection) logistic regression models. These analyses need to be considered as exploratory.

\section{RESULTS}

A radical R0 resection was performed in 74 patients (49.7\%), R1 in $53(35.5 \%), \mathrm{R} 2$ in 21 patients (14.1\%). In addition, one patient $(0.7 \%)$ had no thoracotomy, but underwent remediastinoscopy as previously mentioned.

Clinical (c), tumour (T) and nodal (N) staging before induction chemotherapy versus pathological $(\mathrm{p}), \mathrm{T}$ and $\mathrm{N}$ staging after surgery is depicted in table 2 . The latter is more specifically called ypT and ypN as all these patients had induction chemotherapy. Down-staging to $\mathrm{N} 0$ or $\mathrm{N} 1$ disease was obtained in $40.9 \%$ of patients. 
TABLE 2 Clinical, tumour and nodal (cTN) staging before induction chemotherapy versus pathological, tumour and nodal (ypTN) staging after surgery and induction chemotherapy

T category

N category

\begin{tabular}{|c|c|c|c|c|c|c|c|}
\hline & \multicolumn{4}{|c|}{ ст before induction chemotherapy } & & \multicolumn{2}{|c|}{$\mathrm{cN}$ before induction chemotherapy } \\
\hline & cT1 & сT2 & ст3 & Total & & $\mathrm{cN}_{2}$ & Total \\
\hline урто & 3 & 7 & 1 & $11(7.4)$ & ypNo & 37 & $37(24.8)$ \\
\hline урТ1 & 8 & 24 & 4 & 36 (24.2) & ypN1 & 24 & $24(16.1)$ \\
\hline урТ2 & 4 & 48 & 9 & 61 (40.9) & ypN2 & 84 & 84 (56.4) \\
\hline yртх & 1 & 6 & 2 & $9(6.0)$ & ypNx & 2 & $2(1.3)$ \\
\hline Missing & 0 & 1 & 1 & $2(1.3)$ & Missing & 1 & $1(0.7)$ \\
\hline
\end{tabular}

Data are presented as $n(\%) .{ }^{\#}: n=149$

Operative 30-day mortality was $4.0 \%$ (six patients). Of these patients, five had a pneumonectomy (three on the left and two on the right side), and one a right-sided exploratory thoracotomy. Therefore, 30-day mortality after exploratory thoracotomy occurred in one out of 21 patients $(4.8 \%)$, after lobectomy $0 \%$ and after pneumonectomy five out of 69 patients (7.2\%). Thirty-day mortality after right pneumonectomy was $5.7 \%$ and after left pneumonectomy 9.1\%. One patient died on post-operative day (POD) 4 due to adult respiratory distress syndrome (ARDS), one on POD 5 due to pulmonary hypertension and cardiac decompensation, one on POD 7 because of post-operative bleeding, two on POD 11 due to cardiac decompensation and respiratory insufficiency, and one on POD 26 presumably due to cardiac arrhythmias, although the exact cause of death could not be determined.

Ninety-day mortality was $8.7 \%$ (13 patients), seven patients died between 31 and 90 days post-operatively. Three of these patients had a pneumonectomy, two had a lobectomy, one a bilobectomy and one an exploratory thoracotomy. The causes of death were progressive disease in two patients, infections in two, toxic death in one and other causes in two patients.

In total, 12 patients $(8.1 \%)$ underwent re-operation due to haemothorax (four patients), bronchopleural fistula (five patients), empyema (one patient) and positive margins (two patients).

Regarding the re-operations for haemothorax, in two cases a specific cause could be determined, no precise reason was found in one patient, and the fourth patient had no haemothorax, but a thrombopathy was discovered. Four out of the five bronchopleural fistulas were treated by a Clagett procedure (open window thoracostomy) and one by direct closure of the fistula.

Specific morbidity data was available for 136 patients. These were subdivided into pulmonary, cardiac, pleural space and other complications. The different categories are listed in table 3. Pulmonary complications were most frequent, especially pneumonia and respiratory insufficiency. The observed bronchopleural fistulas all occurred after pneumonectomy. The incidence of this complication after pneumonectomy was $8.7 \%$. Other respiratory complications include mainly cough and dyspnoea due to sputum retention, bronchitis and exacerbation of chronic obstructive pulmonary disease.

Other events in the complications group included urinary tract infection, thrombopathy and uncontrolled diabetes.

\section{TABLE 3 Pulmonary, cardiac, pleural space and other complications of the 136 patients}

\begin{tabular}{lc} 
Pulmonary & $38(27.9)^{\#}$ \\
Atelectasis & 4 \\
Pneumonia & 9 \\
Respiratory insufficiency & 9 \\
Prolonged ventilation & 4 \\
ARDS & 4 \\
Other & 25 \\
Cardiac & $19(14.0)^{4}$ \\
Arrhythmias & 13 \\
Myocardial ischemia & 1 \\
Cardiac decompensation & 5 \\
Pleural space & $25(18.4)^{+}$ \\
Air leak & 12 \\
Empyema & 11 \\
Bronchopleural fistula & 6 \\
Haemothorax & 3 \\
Space problems & 2 \\
Other & $19(14.0)^{5}$ \\
Thromboembolic disease & 2 \\
Wound haematoma & 6 \\
Wound infection & 5 \\
Other & 7 \\
\hline
\end{tabular}

Data are presented as $n$ or $n(\%)$. ARDS: adult respiratory distress syndrome. ${ }^{\#}$ total events $=55 ;{ }^{\top}$ : total events $=19$; $^{+}$: total events $=34$; $^{\text {s. }}$ total events $=20$. 
Investigating the impact of age, sex, histological subtype, cT stage, side of surgery, type of surgery and presence of radical resection on the risk of morbidity or 30-day operative mortality, univariate and multivariate analyses showed that only the type of surgery was significantly associated with occurrence of such an event. Although patients with bilobectomy did not appear to have a significantly different risk of morbidity or mortality $(\mathrm{p}=0.9341)$ than patients with pneumonectomy, patients with exploratory thoracotomy and other surgery did have a significantly lower risk of such an event $(p=0.0048)$ than patients with a pneumonectomy.

\section{DISCUSSION}

In 1994, the EORTC initiated a prospective multicentre trial in which patients with proven N2 disease were initially treated by induction chemotherapy, and subsequently, randomised between surgery and radiotherapy in case of response [2]. The study closed for accrual in December 2002. The primary endpoint was survival. At the time of reporting these results (November 2004), the final results were not yet available, as the number of events allowing statistical analysis had not been reached.

In the present study, the surgical results in the patients randomised to the surgery arm were focused upon. Surgical resection after induction chemotherapy, and especially after chemoradiotherapy, is technically more demanding due to a variable degree of fibrosis that is often encountered after neoadjuvant therapy [8]. This fibrosis renders surgical dissection more tedious, as it is often pronounced at the sites of initial involvement of the different lymph node stations. Therefore, the hilar and mediastinal regions are frequently involved. Frozen section analysis of these lymph nodes can be useful in determining the extent of resection but can be unreliable, especially when only micrometastases persist. When extensive adhesions are present, freeing of the pulmonary artery in the hilum or its branches in the major fissure can be quite difficult, even for an experienced thoracic surgeon, necessitating intrapericardial, retrograde or posterior dissection.

For this reason, it is not surprising that controversial results have been reported on surgical morbidity and mortality after induction chemotherapy or chemoradiotherapy. In the surgery arm of the prospective international multicentre EORTC 08941 trial, mortality was $4.0 \%$ and $8.7 \%$ for operative 30 - and 90 -day, respectively. Morbidity consisted mainly of respiratory $(27.9 \%)$ and pleural space problems (18.4\%).

Older reports already emphasise the higher risk encountered with pneumonectomy in this setting. In a smaller series of 13 patients, FOWLER et al. [4] reported an overall operative mortality of $23 \%$, which increased to $43 \%$ after pneumonectomy. An accompanying editorial cautioned for this excessively high risk [9].

In a large series from the Memorial Sloan-Kettering Cancer Center (New York, USA), 470 patients undergoing lobectomy or pneumonectomy after induction chemotherapy or chemoradiotherapy were retrospectively reviewed [5]. Overall mortality was $3.8 \%, 2.4 \%$ after lobectomy and $11.3 \%$ after pneumonectomy. Right pneumonectomy in this series carried a mortality rate of $23.9 \%$, the specific cause of which could not be determined. Overall morbidity was $38.1 \%$, rising to $58.7 \%$ after right pneumonectomy. Cardiac complications occurred in $12.3 \%$ of the patients and respiratory complications in $25.7 \%$; consisting mainly of pneumonia, respiratory insufficiency, empyema and bronchopleural fistula. Multivariate analysis demonstrated right pneumonectomy, blood loss $>500 \mathrm{~mL}$ and forced expiratory volume in one second $<80 \%$ to be independent risk factors for morbidity; whereas the type of induction therapy had no specific effect [10]. In the EORTC 08941 trial a higher mortality after right pneumonectomy was not observed.

In a series from Houston, USA [11], 76 patients undergoing surgical resection after induction chemotherapy were compared with a series of 259 patients undergoing only surgical resection. Although this was not a randomised study, data on post-operative morbidity and mortality were prospectively collected. Mortality in the surgery-only group was $5.0 \%$ and the combined induction chemotherapy surgery group only $1.3 \%$. However, the number of pneumonectomies was low in this series, being only $10.5 \%$. Major pulmonary complications, including pneumonia, re-intubation, tracheotomy and ARDS, occurred in $13.2 \%$ of patients having surgical resection after induction chemotherapy, but there were no cases of empyema or bronchopleural fistula. The conclusion of the current study was that induction chemotherapy does not significantly affect overall morbidity and mortality of surgical resection. However, it should be emphasised that most patients had a lobectomy or bilobectomy.

Another large series of 350 patients who had surgical resection after induction chemoradiotherapy was reported by STAMATIS et al. [12]. In this retrospective study, 35\% of patients underwent a pneumonectomy and $45 \%$ a lobectomy. Hospital mortality was $4.9 \%, 7.2 \%$ after pneumonectomy, and $3.8 \%$ after lobectomy or bilobectomy. Early or late complications occurred in $44 \%$ of patients, which were mainly cardiac arrhythmias (13\%, 27\% after pneumonectomy), prolonged air leakage $(16 \%)$, pneumonia $(5.1 \%)$, atelectasis $(4.3 \%)$, and septic complications including bronchopleural fistula (4.1\%) and empyema without bronchopleural fistula (1.7\%). Risk factors for pneumonia were high age and a low Karnofsky index, and, for cardiac arrhythmias, abnormal echocardiographic findings prior to surgery. Absence of bronchial stump coverage was another risk factor for peri-operative morbidity. Rethoracotomy for post-operative bleeding was necessary in nine patients $(2.6 \%)$, five after lobectomy and four after pneumonectomy, with a specific cause being found in four patients.

Initial results of the North American Intergroup Trial 0139 investigating the relative role of surgery versus radiotherapy for stage IIIA-N2 NSCLC were recently reported [3]. In contrast to the EORTC study, patients were upfront randomised between a full course of chemoradiotherapy and induction chemoradiotherapy followed by surgical resection. In total, 429 patients were randomised, with 392 being evaluable. In the induction therapy surgery arm, 201 patients participated, with 14 dying from treatment $(6.9 \%)$, four post-operatively and 10 during or after consolidation. The most frequent cause of death was ARDS. There was no difference in 3-yr overall survival between both arms, but progression-free survival was significantly better in the induction therapy surgery group [3]. 
In a very recent study from the University of Pittsburgh, 115 patients who underwent a pneumonectomy from 1990 to 2002 were reported [13]. In total, 50 patients had a right-sided and 65 a left-sided pneumonectomy. Fifty-two patients (45\%) had neoadjuvant therapy, of which 25 had a right and 27 a left pneumonectomy. Median length of stay was 5 days. Right and left pneumonectomies were compared. Thirty-day mortality was $6 \%$ on the right side and $3 \%$ on the left side. Supraventricular arrhythmias were more frequent on the left side, and bronchopleural fistulas more frequent on the right side. Median survival time was not different, being 18.5 months for right-sided and 19.1 months for left-sided interventions. Neoadjuvant therapy increased the risk of postoperative arrhythmias, but not the incidence of bronchopleural fistulas [13].

In conclusion, surgical resection after induction chemotherapy for proven stage IIIA-N2 nonsmall cell ling cancer was found to be feasible in the EORTC 08941 trial, a prospective randomised international multicentre study. In total, six patients died during the first post-operative month, giving a 30-day mortality of $4.0 \%$. Five of the patients who died had a pneumonectomy, three on the left and two on the right side. Morbidity was not very different compared with other large reported series, warranting further studies on combined modality treatment, including surgery for early-stage or locally advanced nonsmall cell lung cancer.

\section{ACKNOWLEDGEMENTS}

The authors would like to thank all participating physicians and institutes of the EORTC Lung Cancer Group who are as follows. The participating physicians from the Netherlands were: B-H. Liem (Lievensberg Ziekenhuis, Bergen Op Zoom); B. Biesma (Bosch Medicentrum-Groot Ziekengasthuis, Hertogenbosch); P. van Valenberg (Elkerliek Ziekenhuis, Helmond); A. Termeer (Canisius-Wilhelmina Ziekenhuis, Nijmegen); T. van Boxem (Ziekenhuis Franciscus, Roosendaal); H. Goey (Maria Ziekenhuis, Tilburg); F. Schramel (St. Antonius Ziekenhuis/Diakonessenhuis, Nieuwegein-Utrecht); A. van Bochove (Ziekenhuis de Heel, Zaandam); P.I. van Spiegel (Slotervaart Ziekenhuis, Amsterdam); N. van Zandwijk (Antoni van Leeuwenhoekhuis, Amsterdam); J. van Meerbeeck /T.A.W. Splinter (Erasmus M.C., Rotterdam); F. van Breukelen (Spaarne Ziekenhuis, Haarlem); V. Tjan-Heijnen (St. Radboud University Hospital, Nijmegen); N.J.J. Schlosser (Universitair Medisch Centrum, Utrecht); G. Giaccone (Academisch Ziekenhuis der Vrije Universiteit, Amsterdam); H.B. Kwa (Onze Lieve Vrouw Gasthuis, Amsterdam); L. Willems (Academisch Ziekenhuis Leiden, Leiden); B.M. Drenth (Atrium Medisch Centrum, Heerlen); J. Stigt (Sophia Ziekenhuis, Zwolle); E. Lammers (Leyenburg Ziekenhuis, Den Haag); G.W.P. Kramer/S. Gans (Arnhem's Radiotherapeutisch Instituut/Ziekenhuis St Jansdal, Arnhem).

The participating physicians from Belgium were: P. Pinson (Universitair Ziekenhuis, Gent); D. van Renterghem (A.Z. St. Jan, Brugge); D. Galdermans (Algemeen Ziekenhuis Middelheim, Antwerpen); P. van Schil (Universitair Ziekenhuis, Antwerpen); L. Delaunois (Cliniques Universitaires de Mont Godinine, Yvoir); J. Vansteenkiste (U.Z. Gasthuisberg, Leuven); M. Beauduin (Hopital de
Jolimont, Haine St Paul); A. Baudoux (Clinique Sainte Elisabeth, Namur); W. Moorkens (AZ Hoge Beuken, Antwerpen); J. Verschuere (AZ Zusters van Barmhasrtigheid, Ronse); J-P. D'Odemont (Clinique Saint Joseph, Mons); W. Verbeke (Stedeljik Ziekenhuis, Aalst).

The participating physicians from Italy were: S. Darwish (Policlinicl Monteluce, Perugia); A. Ardizzoni (Istittuto Nazionale Per la Ricerca Sul Cancro, Genova); G. Scagliotti (Ospedale S. Luigi Gonzaga-Universita di Torino, Torino); and C. Gridelli (Istittuto Nazionale Per lo Studio e la Cura Dei Tumori, Napoli).

The participating physician from Poland was: T. Lewinski (Oncology Centre Institute, Warsaw).

The participating physician from Slovenia was: A. Debeljak (Klinicni Center Ljubljana Golnik, Golnik).

The participating physicians from the UK were: M. Hatton (Weston Park Hospital, Sheffield); M. O'Brien (Royal Marsden Hospital, Sutton); A. Price (Western General Hospital, Edinburgh); and K. O’Byrne (Leicester Royal Infirmary, Leicester).

\section{REFERENCES}

1 Keller SM, Vangel MG, Wagner H, et al. Prolonged survival in patients with resected non-small cell lung cancer and single-level N2 disease. J Thorac Cardiovasc Surg 2004; 128: 130-137.

2 Splinter T, Van Schil P, Kramer G, et al. Randomized trial of surgery versus radiotherapy in patients with stage IIIA(N2) non-small cell lung cancer after a response to induction chemotherapy. EORTC 08941. Clin Lung Cancer 2000; 2: 69-72.

3 Albain KS, Scott CB, Rusch VW, et al. Phase III study of concurrent chemotherapy and full course radiotherapy (CT/RT) versus CT/RT induction followed by surgical resection for stage IIIA( $\mathrm{pN} 2)$ non-small cell lung cancer (NSCLC): first outcome analysis of North American Intergroup trial 0139 (RTOG 93-09). Lung Cancer 2003; 41: Suppl. 2, S4.

4 Fowler WC, Langer CJ, Curran WJ Jr, Keller SM. Postoperative complications after combined neoadjuvant treatment of lung cancer. Ann Thorac Surg 1993; 55: 986-989.

5 Martin J, Ginsberg RJ, Abolhoda A, et al. Morbidity and mortality after neoadjuvant therapy for lung cancer: the risks of right pneumonectomy. Ann Thorac Surg 2001; 72: 1149-1154.

6 Mountain CF. Revisions in the international system for staging lung cancer. Chest 1997; 111: 1710-1717.

7 Van Meerbeeck J, Kramer G, Van Schil P, et al. Induction chemotherapy in stage IIIA-N2 non-small cell lung cancer (NSCLC): an analysis of different regimens used in EORTC 08941. Lung Cancer 2003; 41: Suppl. 2, S79-S80.

8 Liptay MJ, Fry WA. Complications from induction regimens for thoracic malignancies. Perioperative considerations. Chest Surg Clin North Am 1999; 9: 79-95.

9 Rusch VW, Benfield JR. Neoadjuvant therapy for lung cancer: a note of caution. Ann Thorac Surg 1993; 55: 820-821. 
10 Abolhoda A, Martin J, Ginsberg RJ, et al. Morbidity and mortality for pulmonary resections in lung cancer after induction treatment. Lung Cancer 2000; 29: Suppl. 1, 89-90.

11 Siegenthaler MP, Pisters KM, Merriman KW, et al. Preoperative chemotherapy for lung cancer does not increase surgical morbidity. Ann Thorac Surg 2001; 71: 1105-1112.
12 Stamatis G, Djuric D, Eberhardt W, et al. Postoperative morbidity and mortality after induction chemoradiotherapy for locally advanced lung cancer: an analysis of 350 operated patients. Eur J Cardiothorac Surg 2002; 22: 292-297.

13 Patel AN, Luketich JD, Fernando HC, Christie NA, Ferson P, Landreneau RJ. Morbidity of pneumonectomy in the age of neoadjuvant therapy for lung cancer. J Clin Oncol 2004; 22: Suppl. 14, 660s (abstract 7176). 